\title{
LINEAR VS NONLINEAR AND INFINITE VS FINITE: AN INTERPRETATION OF CHAOS
}

\author{
V. Protopopescu
}

DATE PUBLISHED - OCTOBER 1990

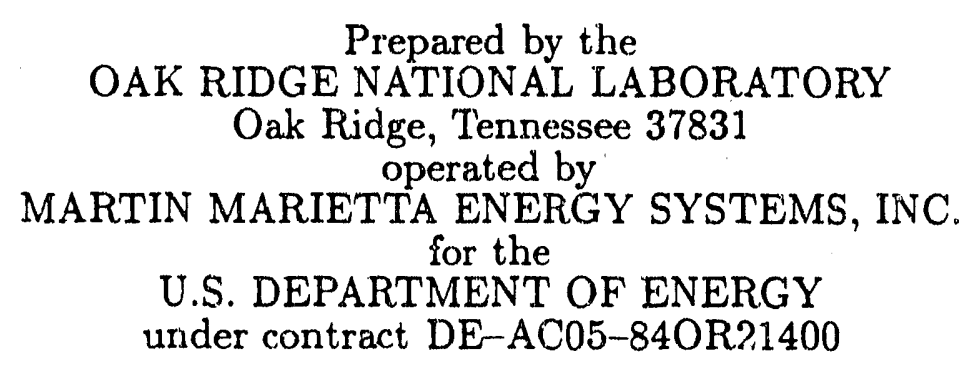




\section{CONTENTS}

ACKNOWLEDGMENTS . . . . . . . . . . . . . . . . v v

ABSTRACT . . . . . . . . . . . . . . . . . . . vii

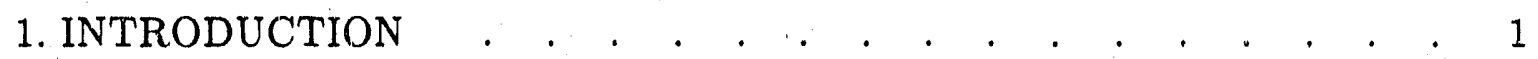

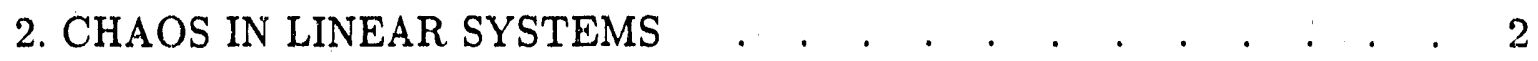

3. RELATIONSHIP WITH NONLINEAR SYSTEMS . . . . . . . . 5

4. INFINITE VS. FINITE

5. CONCLUSIONS . . . . . . . . . . . . . . . . . . . . . . 9

REFERENCES . . . . . . . . . . . . . . . . . . 10 


\section{ACKNOWLEDGMENTS}

This work was partially supported by the U.S. Department of Energy under contract number DE-AC05-84OR21400 with Martin Marietta Energy Systems, Inc. The author acknowledges useful discussions with Professor C. R. MacCluer who made him aware of Ref. 8 . 


\begin{abstract}
An example of a linear infinite-dimensional system is presented that exhibits deterministic chaos and thus challenges the presumably unquestionable connection between chaos and nonlinearity. Via this example, the roles of, and relationships between, linearity, nonlinearity, infinity a finiteness in the occurrence of chaos are investigated. The analysis of these complementary but related aspects leads to: (i) a new interpretation of chaos as the manifestation of incompressible and thus unprocessible information and (ii) a conjecture about the nonexistence of operationally accessible linear systems.
\end{abstract}




\section{INTRODUCTION}

Chaos was thought of by the ancient Greeks as the total absence of structure to the point of emptiness and nonexistence. At the turn of the century, Poincaré intuited it as an unavoidable, inherent threat to mithematical order. Recently, chaos has irrupted in natural sciences and experimental mathematics, to become one of the most fashionable scientific ideas of the 80's. An excellent introduction to the subject, iogether with an extensive bibliography, is provided by Ref. 1. Different descriptors refer to chaos as: (i) stochastic, random; (ii) unpredictable, unstable; (iii) ergodic, mixing; (iv) regular in irregularity; and (v) undecidable. To these qualitative or plainly metaphorical descriptions, there are associated more precise quantitative measures expressed respectively by: (i) power spectra and correlation functions; (ii) Lyapounov exponents; (iii) generated information and Kolmogoroff entropy; (iv) self-similarity and fractal dimensions; and (v) algorithmic complexity. Each of these characterizations captures a specific aspect of chaos as it manifests itself in classical or quantum, conservative or dissipative, continuous or discrete, autonomous or nonau ionomous, deterministic or stochastic systems. ${ }^{1-5}$

In the following, we shall focus on deterministic chaos (i.e., explicitly stochastic sources are excluded) as defined in Devaney's textbook: ${ }^{2}$ if:

Let $X$ be a bounded set. The application $f: X \rightarrow X$ is said to be chaotic on $X$

(a) $f$ is topologically transitive;

(b) the periodic points of $f$ are dense in $X$;

(c) $f$ has sensitive dependence on initial conditions.

This definition implies: (a) indecomposability (almost all orbits fill densely the phase space); (b) a certain element of regularity (there exists a dense set of points that generate periodic orbits): and (c) unpredictability (at least one Lyapounov exponent is positive).

This definition is satisfied by almost all examples dealt with in the literature ranging from turbulence, population interaction, and electrical/mechanical feedback loops to cardiac rhythms, neural activity, and standard numerical schemes (see Refs. 1-5 and references therein).

All the available models that exhibit chaos are nonlinear and conventional wisdom warrants that chaos and nonlinearity are indissolubly related, namely, chaos is assumed to be the manifestation and amplification of the world's inherent nonlinearity. ${ }^{6,7}$ The aim of this paper is to reexamine this assumption and to propose a different interpretation. 


\section{CHAOS IN LINEAR SYSTEMS}

To fix ideas, let $\mathcal{H}$ be an infinite-dimensional separable complex Hilbert space and $\left\{e_{n}\right\}_{n=0,1} \ldots$ an orthonormal basis in $\mathcal{H}$. If $A$ is a bounded operator on $\mathcal{H}$, for any $x \in \mathcal{H}$ we define the orbit of $x$ under the operator $A$ as the sequence $x, A x, A^{2} x, \ldots A^{n} x, \ldots$ If the orbit is dense in $\mathcal{H}$, the vector $x$ is called hypercyclic for the operator $A$. An operator $A$ that has a hypercyclic vector is called hypercyclic (and actually possesses an infinity of hypercyclic vectors). From the definition, it is clear that hypercyclicity is deeply related to condition (a) in Devaney's definition of chaos. In the following, we shall consider that the operator $A$ is linear, i.e. $A(\alpha x+\beta y)=\alpha A x+\beta A y$, for any $\alpha, \beta \in \mathbf{C}$ and any $x, y \in \mathcal{H}$.

The following Lemma is an adaptation of a result of Godefroy and Shapiro, ${ }^{8}$ particularized for the purpose of our presentation.

\section{Lemma}

Let $A: \mathcal{H} \rightarrow \mathcal{H}$ be a linear bounded operator on $\mathcal{H}$ and $Z: \mathcal{K} \rightarrow \mathcal{K} \subset \mathcal{H}$ a (not necessarily linear, not necessarily bounded) operator defined on a dense subset $\mathcal{K}$ of $\mathcal{H}$. Suppose

(i) $A Z=I(I$ is the identity operator and $Z$ is the right inverse of $A)$;

(ii) $\left\{A^{n}\right\}_{n=0,1, \ldots}$ tends to zero on a dense subset of $\mathcal{H}, \mathcal{U}$;

(iii) $\left\{Z^{n}\right\}_{n=0, \ldots}$ tends to zero on a (possibly different) dense subset of $\mathcal{H}, \mathcal{V}$. Then $A$ is hypercyclic.

\section{Proof}

The proof is simply an application of the definition of density of the orbit: namely, starting arbitrarily close from any point $x$, the orbit will wander arbitrarily close to any other point $y$ in the phase space.

For any $x \in \mathcal{U}$, and any $y \in \mathcal{V}$ we have

$$
A^{n} x=\eta_{n} \rightarrow 0, \quad Z^{n} y=\xi_{n} \rightarrow 0
$$

Then

$$
\lim _{n \rightarrow \infty} A^{n}\left(x+\xi_{n}\right)=\lim _{n \rightarrow \infty}\left(A^{n} x+A^{n} Z^{n} y\right)=\lim _{n \rightarrow \infty}\left(\eta_{n}+y\right)=y .
$$

Since $\mathcal{U}$ and $\mathcal{V}$ are dense in $\mathcal{H}$, this concludes the proof. $\diamond$

A concrete realization of the space $\mathcal{H}$ and the operator $A$ from the Lemma is constructed as follows: Let $\mathcal{H}$ be the Hilbert space $l_{2}$ of the infinite sequences $\left(x_{1}, x_{2} \ldots\right)$ such that $\sum_{i=1}^{\infty}\left|x_{i}\right|^{2}<\infty$. An orthonormal basis in this space is provided by

$$
\begin{aligned}
& e_{1}=(1,0,0, \ldots) \\
& e_{2}=(0,1,0, \ldots) \\
& e_{3}=(0,0,1, \ldots)
\end{aligned}
$$

We define the backward shift operator $B: \mathcal{H} \rightarrow \mathcal{H}$ by its action:

$$
\begin{aligned}
& B e_{1}=0 \\
& B e_{n}=e_{n-1}, \quad n>1 .
\end{aligned}
$$


Likewise, we define the forward shift $F: \mathcal{H} \rightarrow \mathcal{H}$ by

$$
F e_{n}=e_{n+1} \quad n \geq 1 \text {. }
$$

The operators $B$ and $F$ are obviously linear operators on $\mathcal{H}$. The spectrum of $B, \sigma(B)$ is the whole unit disk $\sigma(B)=\{\mu \| \mu \mid \leq 1\}$. Indeed, for any complex number $\mu$ inside the unit disk, the vector $e_{1}+\mu e_{2}+\mu^{2} e_{3}+\ldots$ belongs to $\mathcal{H}$ since its norm is bounded by $\left(1-|\mu|^{2}\right)^{-1}<\infty$ and is an eigenvector of $B$ with eigenvalue $\mu$ as verified by the equality

$$
B\left(e_{1}+\mu e_{2}+\mu^{2} e_{3}+\ldots\right)=\mu\left(e_{1}+\mu e_{2}+\ldots\right) .
$$

The circle $|\mu|=1$ belongs to the spectrum by the closure property. The outside of the disk belongs to the resolvent set of $B$, since $\|B\|=1$ and thus for any $|\lambda|>1$, the operator $\lambda I-B$ is boundedly invertible.

The following result holds true:

Proposition

If $B$ is the backward shift defined in the previous section, the linear map $A \stackrel{\text { def }}{=} \gamma B: \mathcal{H} \rightarrow \mathcal{H},|\gamma|>1$ exhibits the properties (a), (b), (c), required as signatures of deterministic chaos.

Proof

The proof of property (a) has been first given by Rolewicz ${ }^{9}$ and simplified by Godefroy and Shapiro who set it in a more general and natural context. ${ }^{8}$ Trivial calculations yield properties (b) and (c).

(a) In order to verify the hypercyclicity, we show that $A$ satisfies the hypothesis in the Lemma above. We define the operator $Z \stackrel{\text { def }}{=} \gamma^{-1} F$ where $F$ is the forward shift. Then

$$
\begin{aligned}
& A Z=(\gamma B)\left(\gamma^{-1} F\right)=I, \\
& \left\|Z^{n}\right\|=\left\|\left(\gamma^{-1} F\right)^{n}\right\| \leq \frac{\left\|F^{n}\right\|}{|\gamma|^{n}}=\frac{1}{|\gamma|^{n}} \rightarrow 0, \\
& \left\|A^{n}\right\|=\left\|(\gamma B)^{n}\right\| \text { tends to zero by the action of } B .
\end{aligned}
$$

Thus, by the Lemma above, the operator $A$ is hypercyclic.

(b) Not all hypercyclic operators are chaotic, but we can show that the operator $A$ has dense periodic orbits. Indeed, the spectrum of $A, \sigma(A)$, is the disk $\{\gamma \mu \| \mu \mid \leq 1\}$ and contains the unit circle. The unit circle is densely filled with numbers of the form $\kappa=\exp (2 \pi i m / n), m, n \in \mathbf{Z}$, and each of these numbers is a discrete eigenvalue of $A$ corresponding to the eigenvector $e_{1}+$ $\frac{\kappa}{\gamma} e_{2}+\frac{\kappa^{2}}{\gamma^{2}} e_{3} \ldots$ Linear combinations of this form are dense in the space $\mathcal{H}$.

(c) To prove that the system exhibits a positive Lyapounov exponent, we compute the long-time effect of the evolution on two initially neighboring vectors $x_{0}$ and $x_{1} \stackrel{\text { def }}{=} x_{0}+g, g=\epsilon\left(e_{1}+\mu e_{2}+\ldots\right), \epsilon$ small, $1 .>|\mu|>|\gamma|^{-1}$. The Lyapuunov exponent is defined as ${ }^{2}$

$$
\lambda=\lim _{n \rightarrow \infty} \frac{1}{n} \log \frac{\left\|(A)^{n} x_{1}-(A)^{n} x_{0}\right\|}{\left\|x_{1}-x_{0}\right\|}=
$$




$$
\lim _{n \rightarrow \infty} \frac{1}{n} \log \frac{\left\|(A)^{n} g\right\|}{\|g\|}=\lim _{n \rightarrow \infty} \frac{1}{n} \log |\gamma \mu|^{n}=\log |\gamma \mu|
$$

and thus for our choice of $\mu$ turns out to be positive. $\diamond$

Remark.

The result of the Proposition remains true for any operator $f(B)$ whose spectrum contains a portion of the unit circle. The particular example $f(B)=e^{B}$ has been presented in Ref. 10. The proofs for all these mappings are simple corollaries of Godefroy and Shapiro's result. 


\section{RELATIONSHIP WITH NONLINEAR SYSTEMS}

The result contained in the Proposition above seems puzzling and counterintuitive, but a closer inspection shows that it should not be that surprising.

First, it is well-known ${ }^{1,2}$ that via the nonlinear transformation

$$
x_{n} \stackrel{\text { def }}{=} \sin ^{2} \pi y_{n},
$$

the logistic map

$$
x_{n+1}=\rho x_{n}\left(1-x_{n}\right)
$$

with $\rho=4$ (i.e., in full-blown chaotic regime) is equivalent with the transformation

$$
y_{n+1}=\mathcal{B}\left(y_{n}\right) \stackrel{\text { def }}{=} 2 y_{n}(\bmod 1) \text {. }
$$

This map realizes a nonlinear and noninvertible transformation of the segment $[0,1]$ into itself. We can regard this example in the following way: on the segment $\mathcal{X} \stackrel{\text { def }}{=}$ $[0,1]$, with the point one identified with zero, we introduce the mod 1-addition, $\oplus: \mathcal{X} \rightarrow \mathcal{X}$. The set $\mathcal{X}$ is a group under this operation, with zero as the identity element. We introduce also the exterior mod 1-multiplication with real scalars, $\otimes: \mathbf{R} \times \mathcal{X} \rightarrow \mathcal{X}$, but this multiplication does not have the required properties to transform $\mathcal{X}$ into a linear space, since in general, for $x \in \mathcal{X}, \alpha, \beta \in \mathbf{R}, \alpha \otimes(\beta \otimes x) \neq$ $(\alpha \beta) \otimes x$. If we write the numbers in $\mathcal{X}$ in binary representation, the nonlinear operator $\mathcal{B}$ acts like a backward shift, namely, the whole string of digits moves left one position and the leftmost digit is chopped off. In this representation, the operator $\mathcal{B}$ is known as the Bernoulli shift ${ }^{1}$ and has al! the properties required by chaos since: (i) if $x$ is irrational, the orbit fills densely the interval $\mathcal{X}$; (ii) if $x$ is rational, $\mathcal{B}$ yields periodic orbits; and (iii) the Lyapounov exponent of this map is $\log 2>0$. Although there is no linear structure preserved here, the Bernoulli shift $\mathcal{B}$ is formally similar to the backwird shift $B$ introduced in the previous section.

Second, there exists a deeper, rather general correspondence between finitedimensional nonlinear maps (equations) and infinite-dimensional linear maps (equations), that was pointed out by Carleman almost 60 years ago. ${ }^{11}$ Following an idea of Poincaré, Carleman showed that there is a natural embedding of finite-dimensional systems of ordinary differential equations into infinite systems of linear differential equations. This approach is known today as Carleman linearization or Carleman embedding. This embedding can be extended to maps, as was shown by Steeb. ${ }^{12}$ For instance, in the case of the logistic map mentioned before. the definitions $\left(x_{n}\right)^{k} \stackrel{\text { def }}{=} v_{k, n}$ transform the finite dimensional nonlinear map into ine infinite dimensional linear system

$$
\begin{aligned}
& v_{1, n+1}=\rho\left(v_{1, n}-v_{2, n}\right) \\
& v_{2, n+1}=\rho^{2}\left(v_{2, n}-2 v_{3, n}+v_{4, n}\right)
\end{aligned}
$$

Vice-versa, the linear iteration map

$$
x_{n+1}=B x_{n}
$$


induced by the backward shift $B$ on the infinite-dimensional space $\mathcal{H}$, introduced in the previous section, corresponds to a nonlinear one-dimensional transformation. Indeed, at the $\mathrm{n}$-th iteration the action of the operator $B$ can be written component wise as

$$
\begin{aligned}
& x_{1, n+1}=x_{2, n} \\
& x_{2, n+1}=x_{3, n}
\end{aligned}
$$

On the subset of vectors $x \in \mathcal{H}$ whose components are restricted to the set $\{0,1\}$, the backward shift $B$ acts in $\mathcal{H}$ exactly as the Bernoulli shift $\mathcal{B}$ acts in $\mathcal{X}$. Thus, for this subset of vectors (which is not a linear subspace of $\mathcal{H}$, but only a subgroup) the linear transformation above reads

$$
x_{n+1}=2 x_{n}(\bmod 1)=2 \otimes x_{n}
$$

where now $x_{n}$ can be viewed as a real number in $\mathcal{X}$. For a general vector in $\mathcal{H}$, the nonlinear transformation corresponding to the linear map $B$ is not easy to explicitate.

Th? puzzling aspect of "chaos in linear systems" really dissipates if one realizes that while the backward shift $B$ acts linearly as an operator on $\mathcal{H}$, it acts nonlinearly as an operation on the individual components of the vectors in $\mathcal{H}$. Actually, this operation depends entirely on the vector itself. Since almost all the vectors in $\mathcal{F}$ (like the irrational numbers in $\mathcal{X}$ ) contain an infinite (incompressible) amount of information, it is only natural that trouble will arise at any finite operational level, unable to process an infinite amount of information.

\section{Remarks.}

1.Nonlinear equations are not necessary, in principle, to describe nonlinear phenomena: the most intricated situations in Hamiltonian classical mechanics and quantum many body theory can be described by the linear Liouville and Schrodinger equations, respectively. Nonlinearities usually appear when one projects (reduces) the complete dynamical information contained in the solutions of these equations onto the actically observable and/or physically interesting quantities.

2.The Carleman linearization is different from both the local (trivial) linearization of a nonlinear equation around a given solution and the global linearization of nonlinear equations via suitably chosen nonlinear function transformations (e.g. the Hopf- Cole transformation that takes the Burgers equation into the heat equation.) ${ }^{1}$

3.The backward shift $B$ on which the previous construction is based is not a farfetched example. On the contrary, the shift is a fundamental "building block" of linear operators on Hilbert spaces. Indeed, let $A$ be any bounded operator on such a space; then $A /\|A\|$ is a contraction. Likewise, for almost any unbounded operator $A$, there exist $\alpha, \beta \in \mathbf{C}$ such that $e^{\alpha A+\beta}$ is a contraction. Any contraction can be canonically lifted to an isometry, and any isometry is uniquely decomposable into a unitary operator and a shift. (For precise statements and proofs see Ref. 13.) Thus, the importance of the example presented in Section 2 may run deeper than a cute exercise. 


\section{INFINITE VS. FINITE}

From the examples before, it appears that chaos does not emerge from nonlinearity per se, but seems to be connected with the pathologies of infinity and with our inadequacy of handling infinite amounts of information.

It is well-known that: ${ }^{14-16}$ (i) a single irrational number exceeds the capabilities of our past, present, and future operational capacity, (ii) most numbers are irrational, so in most situations we shall be confronted with their "chaotic" features, (iii) the question whether a number is irrational or not is, in general, undecidable, and (iv) most irrational numbers are uncomputable, i.e. they cannot be computed by an algorithm shorter than the digit string itself. Due to the finiteness of our lives and the finiteness of our computers' precision, we are bound to operate with finite arithmetic. Within finite arithmetic, the equivalence mentioned before between infinite linear systems and finite nonliness systems is lost: if the computer accepts only $N$ digit binary strings, the Bernoulli shift $\mathcal{B}$ will transform every number into zero after $N$ steps, while the "equivalent" logistic map will continue to yield numbers different from zero.

Deterministic (linear or nonlinear) systems on finite arithmetic deterministic computers lead only to fixed points and/or (highly) periodic orbits. ${ }^{17}$ Two questions are then, in order:

1) What exactly do/can we compute?

2) Does it make sense to look for deterministic chaos?

The answer to the first question is that: on one hand, we compute average (statistic) or global properties, such as Lyapounov exponents or fractal dimensions, that, due to the structural stability of most dynamical systems are not sensitive to small uncertainties; on the other hand, if one follows the individual dynamics, one is forced by the finite arithmetic to do it only in a coarse-grained sense that yields at most highly periodic orbits.

The answer to the second question is more elusive. In order to separate the effects of the numerical truncations from those of the dynamics itself, Rannou ${ }^{18}$ studied the integer mapping

$$
\begin{aligned}
& x_{n+1}=x_{n}+y_{n}+\left[\frac{m}{2 \pi}\left(1-\cos \frac{2 \pi y_{n}}{m}\right)\right] \quad(\bmod m) \\
& y_{n+1}=y_{n}-\left[\frac{K m}{\pi}\left(\sin \frac{2 \pi x_{n+1}}{m}+1-\cos \frac{2 \pi x_{n+1}}{m}\right)\right] \quad(\bmod m)
\end{aligned}
$$

where the square brackets, $[\alpha]$, denote the integer nearest to $\alpha$ and $K$ is the stochasticity parameter (analog to $\rho$ in the logistic map). The initial values $x_{0}, y_{0}$ are integers between $-m / 2$ and $m / 2$ and by the integer mapping above so will be $x_{n}, y_{n}$. Depending on the values of $K$, this integer mapping displays shorter or longer cycles that, in some regimes, visit rather uniformly the $m^{2}$ points of the lattice. With $m$ of the order of 1,000 , relatively long cycles and uniform fillings do indeed occur.

In order to investigate the "randomness" of the deterministic integer mapping above, Rannou carrieci out a parallel study by assigning the probability $1 /\left(m^{2}\right)$ ! to each of the $\left(m^{2}\right)$ ! possible mappings of $m^{2}$ points into $m^{2}$ points and examining the output of this random mapping. He found that: 
- the probability of occurrence of a cycle of length $n$ is $1 / m^{2}$, independent of $n$ and of the initial point, and

- the average length of a cycle is $\left(m^{2}+1\right) / 2$.

He noticed good agreement between these properties and the properties of the deterministic map in the apparently random regime.

So, the question arises anew: Is chaos intrinsic to dynamics after all? Today's best answer is that we simply don't know and we don't seem to be more able than the ancient Greeks to fully deal with infinity and irrationality - at least not at the operational level.

A possible answer is that randomness in physical systems is related to NPcompleteness of the relative problem, i.e. to computational irreducibility, numerical complexity, and undecidability. ${ }^{16}$

We also advance the conjecture that, in a true sense, linear systems cannot exist but within an infinite world (and thus are operationally irrelevant) and any type of constraints imposed by tise finiteness of our operational world (e.g. boundary conditions, numerical truncations, etc.) always introduces nonlinearities. In this sense, one can say that chaos is related to nonlinearity. 


\section{CONCLUSIONS}

We have addressed the question concerning the origin of chaos and presented an example for which we could find definite and sometimes puzzling answers to this question. Linear and nonlinear are somewhat interchangeable features, depending on scale and representation. Chaos is nut generated by nonlinearity per se, but by infinity. Finits-dimensional nonlinear systems may exhibit chaos as a reflection of the underlying (linear) infinite-dimensional world. In other words, chaotic behavior occurs only when we have to deal with infinite amounts of information at a finite level of operability. In this sense, even the most deterministic system will behave stochastically due tc inavoidable and unknown truncations of information.

Mathematical deiurminism does not always imply physical/observational determinism. Physical in.picdictability arises from the impossibility of (i) making physical measureinents with infinite precision and (ii) operating with real numbers within infinite precision. Even when, like in Rannou's integer mapping, these problems are discarded and the cvolution is completely predictable, both mathematically and experimentally, it may happen that for some values of $K$ the output becomes too complicuted, too cumbersome to grasp and operate with, and therefore finally irrelevant. The same thing would happen if we were able to know initial conditions with infinite precision and solve $10^{23}$ equations in a second. We would not want to know $10^{23}$ velocities at any given time, but rather use thern to calculate their quadratic mean, i.e. the actual value of the kinetic energy. Up to very small fluctuations, this would be precisely a projected quantity of statistical nature - namely the temperature. In suc'. instances, the specificity of our epistemologic paradigm and tine human mind's rather synthetic way of accessing, processing, and transferring information makes statistical descriptions preferable to exact descriptions. 


\section{REFERENCES}

1. E. Atlee Jackson, Perspectives of Nonlinear Dynamics, Cambridge University Press, Cambridge, Vol. 1, 1989, Vol. 2, 1990.

2. R. L. Devaney, An Introduction to Chaotic Dynamical Systems, The Benjamin Cummings Publishing Co., Inc., Menio Park, C 4, Iỹoj.

3. A. V. Holden, ed., Chaos, Princeton University Press, Princeton, 1986.

4. P. Bergé, Y. Pomeau, and C. Vidal, Order Within Chaos, John Wiley \& Sons, New York, 1984.

5. J. M. T. Thompson and H. B. Stewart, Nonlinear Dynamics and Chaos, John Wiley \& Sons, New York, 1986.

6. S. Neil Rasband, Chaotic Dynamics of Nonlinear Systems, John Wiley \& Sons, New York, 1990.

7. S. Eubank and D. Farmer, "An Introduction to Chaos and Randomness," Los Alamos National Laboratory (LANL) Preprint, LA -UR 90-1874.

8. G. Godefroy and J. S. Shapiro, "Operators with Dense, Invariant, Cyclic Vector Manifolds," J. Funct. Anal. (1990).

9. S. Rolewicz, "On Orbits of Elements," Studia Math. 33, 17-22 (1969).

10. C. R. MacCluer, "Chaos in Linear Distributed Systems," in Proceedings of the 29th IEEE Conference on Decision and Control, Honolulu, Hawaii, December, 1990.

11. T. Carleman, "Application de la Théorie des Equations Integrales Linéaires aux Systèmes d'Equations Differentielles Nonlinéaires," Acta Math. 59, 63-87 (1932) (in French).

12. W.-H. Steeb, "Linearization Procedure and Nonlinear Sy" tems of Differential and Difference Equations," in Nonlinear Phenomena in Chemical Dynamics, Ecis. C. Vidal and A. Pacault, Springer Verlag, Berlin, 1981, p. 275.

13. B. Sz.-Nagy and C. Foiaş Analyse Harmonique des Opérateurs de l'Espace de Hilbert, Masson et Cie, Ahiadémiai Kiadó, Budapest, 1967.

14. J. Ford, "How Random is a Coin Toss," Physics Today, April, 40-47 (1983).

15. R. V. Jensen, "Classical Chaos," Arnerican Siientist, 75, 168-181 (1987).

16. C. Agnes and M. Rasetti, "Chaos and Undecidability: A Group Theoretical View," in 10th Annual Open "Iniversity Conference on Statıstical Mechanics, World Scientific, Teaneck, NJ, 1988, pp. 57-73.

17. P. M. Binder and R. V. Jensen, "Simuls'ing Chaotic Behavior with Finite-Staie Machines," Phys. Rev. A 34, 4460-A 463 (1986).

18. F. Rannou, "Numerical Study of Discrete Plane Area-Preserving Map," Astron. Astroph. 31, 289-301 (1974). 

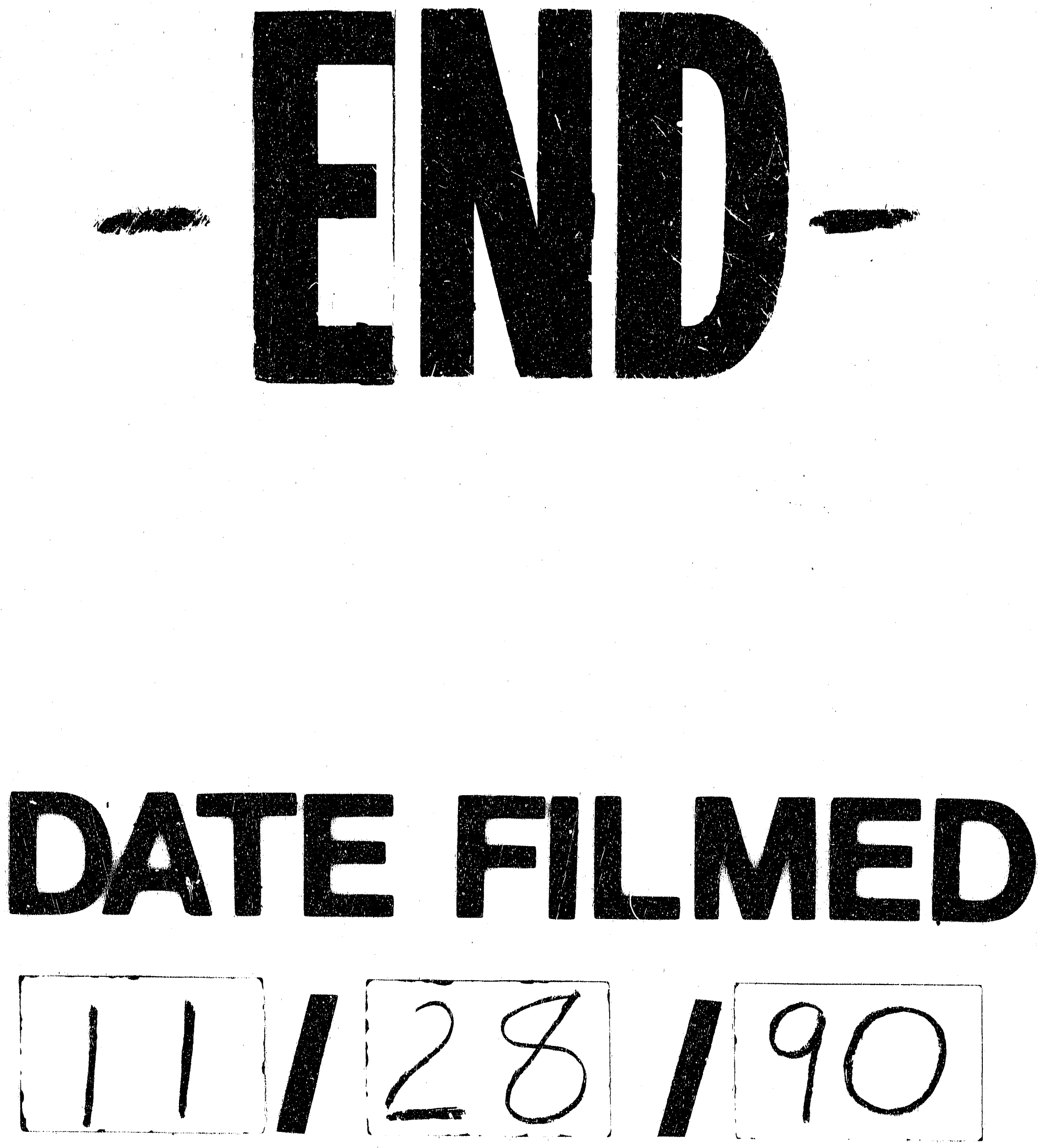
